$9-7-2021$

\title{
Atención de la salud sexual y reproductiva de mujeres migrantes: Un mapeo de actores de la sociedad civil en la Ciudad de México-2020
}

Silvana Larrea-Schiavon

Population Council

Lucía Vázquez Quesada

Population Council

Esperanza Basurto-Alcalde

Natalia Polgovsky

Isabel Vieitez Martínez

Population Council

See next page for additional authors

Follow this and additional works at: https://knowledgecommons.popcouncil.org/departments_sbsr-rh How does access to this work benefit you? Let us know!

\section{Recommended Citation}

Larrea-Schiavon, Silvana, Lucía Vázquez-Quesada, Esperanza Basurto-Alcalde, Natalia Polgovsky, Isabel Vieitez Martínez, and Pooja Sripad. 2021. "Atención de la salud sexual y reproductiva de mujeres migrantes: Un mapeo de actores de la sociedad civil en la Ciudad de México-2020." México: Population Council. 


\section{Authors}

Silvana Larrea-Schiavon, Lucía Vázquez Quesada, Esperanza Basurto-Alcalde, Natalia Polgovsky, Isabel Vieitez Martínez, and Pooja Sripad 


\section{Atención de la salud sexual y}

reproductiva de mujeres migrantes: Un mapeo de actores de la sociedad civil en la Ciudad de México

\section{0}

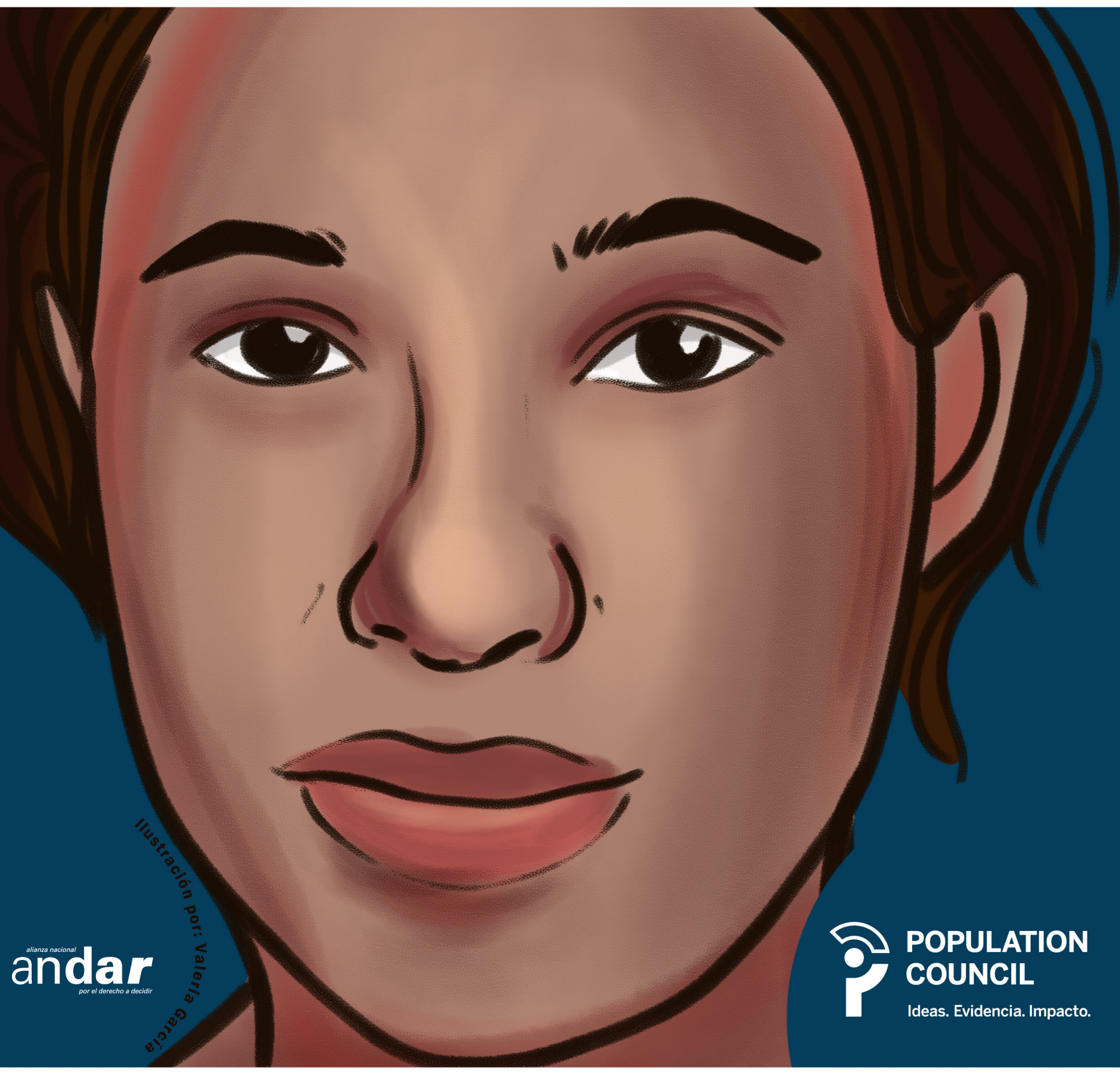




\section{Popvuatoon COUNCIL}

Ideas. Evidencia. Impacto.

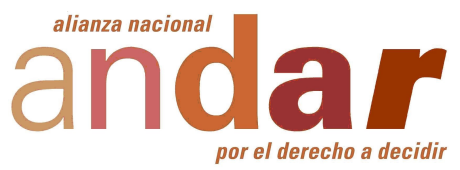

\section{Atención de la salud sexual y reproductiva de mujeres migrantes: Un mapeo de actores de la sociedad civil en Ciudad de México, México. 2020.}

\section{Equipo de investigación:}

Protocolo de investigación, análisis de datos, redacción y/o revisión de informe:

Silvana Larrea-Schiavon* Lucía Vázquez-Quesada* Isabel Vieitez Martínez*

Pooja Sripad*

*Population Council.

Coordinación del proyecto, recolección y análisis de datos, redacción y revisión de informe:

Esperanza Basurto-Alcalde, consultora.

Recolección de datos, análisis de datos, y redacción de informe:

Natalia Polgovsky-Ezcurra, consultora.

Recolección de datos (personas pasantes y voluntarias):

Arturo Alamilla, entrevistador. Ana Regina Nájera, entrevistadora. Ximena Nateras, entrevistadora. Iván Coria, entrevistador. Gemma Hernández, entrevistadora.

\section{Corrección de estilo:}

Claudia Martínez.

\section{Ilustración y diseño editorial:}

Valeria García.

\section{Agradecimientos:}

Agradecemos el esfuerzo realizado por el equipo de investigación, sobre todo a personas pasantes y voluntarias, así como la gran disposición que las personas entrevistadas tuvieron para compartirnos su experiencia, en pleno inicio de la pandemia causada por el virus SARS-CoV-2, y por su compromiso constante con las mujeres migrantes y otras poblaciones en movilidad.

\section{Aviso legal:}

Se permite la reproducción total o parcial de esta publicación para fines educativos, de investigación y cualquier otro fin libre de lucro, siempre y cuando se cite la fuente.

\section{Cita sugerida:}

Larrea-Schiavon Silvana, Vázquez-Quesada Lucía, Basurto-Alcalde Esperanza, Polgovsky Natalia, Vieitez Isabel, and Sripad Pooja. 2021. "Atención de la salud sexual y reproductiva de mujeres migrantes: Un mapeo de actores de la sociedad civil en Ciudad de México, México. 2020." México: Population Council. 


\section{HALLAZGOS PRINCIPALES}

- La Ciudad de México (CDMX) se declaró ciudad santuario en 2017. Las personas migrantes suelen permanecer en la Ciudad por más tiempo que en otros estados; solicitan asilo y buscan empleo temporal.

- Desde 2007, la Interrupción Legal del Embarazo (ILE) se permite dentro de las primeras 12 semanas de gestación. Es un servicio gratuito y se presta en clínicas especializadas de primer nivel y hospitales asignados.

- La capital concentra las sedes de organizaciones internacionales y nacionales que trabajan en la defensa de los derechos humanos.

- Se realizó un total de 14 entrevistas a organizaciones de la sociedad civil (OSCs), de las cuales 12 son laicas, y sólo 3 trabajan la intersección entre género y población migrante.

- La canalización es el servicio más brindado en todas las temáticas de salud sexual y reproductiva (SSyR) exploradas (sobre todo el parto); seguido de información sobre anticoncepción y consejería sobre ILE. Pocas organizaciones brindaron atención directa (1 ha atendido parto con parteras, y 3 dieron atención directa para anticoncepción, anticoncepción de emergencia, e ILE).

- Las barreras para el acceso a servicios de SSyR que se mencionaron son normativas (confusión sobre el funcionamiento del Insabi); institucionales (racismo, xenofobia, atención discrecional, y falta de recursos e insumos); e individuales (desconocimiento de derechos, tabús sobre la SSyR, machismo, e idioma para poblaciones que no hablan castellano).

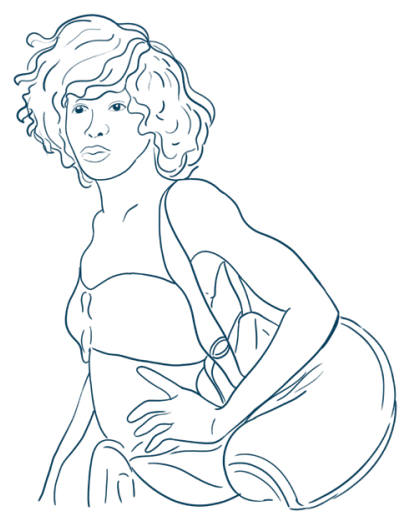

Nota de campo Si van referidas, no hay problema en atenderlas, pero si van por su propio pie, no les han querido dar la atención.

-Organización, Ciudad de México
- La COVID-19 ha incrementado las barreras de acceso a servicios de salud, incluida la SSyR; y ha sido un reto para la labor de las organizaciones, que muchas veces dependen de trabajo voluntario para su funcionamiento y no necesariamente estaban preparadas para una contingencia sanitaria.

- La promoción de los servicios de las OSCs se da de boca en boca y por referencias directas de otras organizaciones con quienes tienen acuerdos.

\section{INTRODUCCIÓN}

Con el respaldo de la Constitución Política de la Ciudad de México ${ }^{1}$ (2017) la CDMX se declaró ciudad santuario en 2017, es decir, ciudad de acogida y de garantía de derechos (techo, alimentación, salud) a las personas que llegan a la entidad, independientemente de su estatus migratorio y razones por las que migraron (Oxfam, 2018). También se facilita el estudio y la inserción laboral a través de programas específicos para ello.

La Comisión Mexicana de Ayuda a Refugiados (Comar) y el Alto Comisionado de las Naciones Unidas para los Refugiados (ACNUR) son actores clave en el tema de migración, y ambos tienen su sede en la Ciudad de México. Estos dos organismos canalizan a las personas migrantes solicitantes de asilo a las organizaciones de la sociedad civil que pueden ofrecer un espacio de alojamiento, asesoría, y acompañamiento para el acceso a sus derechos, incluido el derecho a la salud. Por tanto, las personas migrantes indocumentadas permanecen relativamente más tiempo en la ciudad capital: solicitan asilo y/o buscan trabajo temporal (ACNUR, 2017). De las mujeres solicitantes de asilo en $2018(n=7,112)$, el 68\% fueron centroamericanas (Comar, 2018). El Diagnóstico y Programa de Derechos Humanos de la Ciudad de México (2016) estipula el derecho a la salud independientemente del estatus migratorio.

Por último, en la Ciudad de México, la interrupción del embarazo a demanda es legal dentro de las primeras 12 semanas de gestación, y existen clínicas especializadas para esta atención. Otras causales de exclusión de responsabilidad por aborto son alteraciones congénitas, inseminación artificial no consentida, riesgo para la salud de la persona gestante, y cuando el embarazo es producto de violencia sexual -en estos casos, las semanas de gestación en que se puede realizar la interrupción del embarazo es mayor a las 12 semanas-. 


\section{OBJETIVO Y METODOLOGÍA}

El presente estudio se enfocó en identificar si las organizaciones de la sociedad civil brindan los servicios de difusión de información, consejería, canalización, acompañamiento y atención directa a mujeres migrantes en tránsito por México en temas referentes a métodos anticonceptivos, anticoncepción de emergencia, interrupción del embarazo, pruebas de embarazo, atención prenatal, atención del parto, atención del postparto, salud menstrual, infecciones de transmisión sexual (ITS) y atención a sobrevivientes de violencia sexual.

Para alcanzar dicho objetivo se hizo una búsqueda dirigida de organizaciones que trabajaran en temas de SSyR y/o con mujeres migrantes en siete estados claves en la ruta migratoria en la República mexicana: Chiapas, Tabasco, Veracruz, Oaxaca, Ciudad de México, Chihuahua y Baja California. Se identificó a un actor clave dentro de cada organización y se realizó una entrevista telefónica semiestructurada (para más información ver el reporte general del proyecto). En este documento se presentan los resultados para la Ciudad de México.

\section{RESULTADOS}

\section{Perfil de las organizaciones entrevistadas}

Por búsqueda dirigida se identificó a un total de 42 organizaciones que cumplían con los criterios de inclusión, de las cuales se logró entrevistar a 14 (33\%): 10 organizaciones nacionales, 3 internacionales y 1 fundación. Dentro de estas OSCs, 7 prestan servicios de estancia-refugio y asistencia humanitaria; y las otras 7 hacen trabajo de incidencia. Asimismo, 12 son laicas, y 9 tienen como población objetivo la migrante, mientras que 5 trabajan más particularmente temas de SSyR (aunque han atendido población migrante).

\section{Servicios brindados para la atención de la} SSyR de las mujeres migrantes

Del total de organizaciones entrevistadas en la Ciudad de México, 14 (100\%) difunden información, 12 (86\%) dan consejería, 14 (100\%) canalizan casos, 13 (93\%) brindan acompañamiento para la atención (2 sólo en casos específicos); y 11 (79\%) brindan atención directa ${ }^{2}$ en alguno de los temas mencionados.

Cuando se hace el cruce entre tipo de servicio (difusión de información, consejería, atención directa, canalización y referencia y acompañamiento) y temática de SSyR en la cual se ofertó este servicio, se identifica que los principales temas sobre los cuales se difundió información en las organizaciones fueron la atención a mujeres que han vivido violencia sexual, métodos anticonceptivos, ITS, interrupción del embarazo y salud menstrual. Con respecto a los principales temas sobre los cuáles se brindó consejería, se identificaron la atención a mujeres que han vivido violencia sexual, interrupción del embarazo, ITS, cuidados maternos (atención prenatal, atención del parto), y anticoncepción de emergencia. La atención directa brindada por las organizaciones fue para mujeres que han vivido violencia sexual.

La canalización y referencia fue el servicio que se brindó por la mayoría de las organizaciones en casi todos los temas (más del 70\%, excepto para la salud menstrual). Por último, el acompañamiento se brindó a mujeres que vivieron violencia sexual y en las temáticas relacionadas al embarazo (prueba, interrupción, atención prenatal y parto) (ver Tabla1).

La información se brindó principalmente a través de sinergias entre las organizaciones especialistas en SSyR y las que ofrecen estancia a migrantes; y se hizo a través de materiales, pláticas o talleres de acuerdo con las posibilidades y coyunturas. Es decir, no parece ser un servicio constante, y destaca la insuficiente información y atención de necesidades de salud menstrual, anticoncepción (incluida la de emergencia), prueba de embarazo, y cuidados prenatales. En los distintos temas de salud reproductiva, la consejería se entiende como un servicio de orientación sobre dónde pueden encontrar más información o a qué organización o centro de salud pueden referirse para su atención. Las canalizaciones se hicieron a otras OSCs o a los sistemas públicos de salud; sin embargo, según el reporte, sin acompañamiento de las organizaciones, el servicio público es negado a las mujeres migrantes.

La atención a víctimas de violencia sexual fue el servicio más brindado (sobre todo a través de apoyo psicológico), pero son pocas las organizaciones que manejan anticoncepción de emergencia, profilaxis postexposición para $\mathrm{VIH}$, y pruebas de embarazo. Los servicios relativos a ITS se restringen generalmente a $\mathrm{VIH}$, y no es claro qué acciones se toman sobre información y tratamiento de otras infecciones. También resalta que la información, consejería y referencia sobre interrupción del embarazo la brindaron principalmente organizaciones feministas que han trabajado o trabajan con migrantes, pero cuya población objetivo es más amplia. A las mujeres embarazadas se les brindó referencia y acompañamiento, sobre todo para acceder a servicios

${ }^{2}$ De éstas, 1 afirmó brindar atención directa pero no médica; y 1 más dijo brindar atención directa con el apoyo de muchas organizaciones. 
de salud para el parto. La única organización que reportó brindar atención a partos dijo hacerlo en 3 casos y que se han apoyado en parteras -a solicitud de las mujeres migrantes-.

En conjunto y recurrentemente, las organizaciones enfatizan la imposibilidad que tienen de brindar atención médica directa en salud y SSyR, así como la responsabilidad que tiene el estado de hacer valer ese derecho para todas y todos, incluida la población migrante. Aunque no lo mencionaron todas las OSCs entrevistadas, los consultorios adyacentes a las farmacias parecen ser un punto de contacto de las y los migrantes con el sistema de salud privado de bajo costo.
Pese a no tener información de una organización al respecto, 10 OSCs no cuentan con personal de salud, 3 dijeron sí tenerlo (ver Gráfica 1). Las que cuentan con personal médico, también tienen personal de enfermería y/o trabajo social o en promoción de la salud. Varias OSCs enfocan su trabajo a la incidencia, por lo que es entendible que no cuenten con personal médico. Posiblemente, la concentración de servicios de salud en la capital del país reduce la necesidad (o prioridad, dados los recursos limitados de las organizaciones) de tener personal de salud de base; $y$ hace posible la exigibilidad del derecho a la salud.

\begin{tabular}{|c|c|c|c|c|c|c|}
\hline & & $\begin{array}{l}\text { Difusión de } \\
\text { información }\end{array}$ & Consejería & $\begin{array}{l}\text { Atención } \\
\text { directa }\end{array}$ & $\begin{array}{l}\text { Canalización y } \\
\text { referencia }\end{array}$ & Acompañamiento \\
\hline & Métodos anticonceptivos n (\%) & $8(57)$ & $4(29)$ & $3(21)$ & $10(71)$ & $1(7)$ \\
\hline & Infecciones de transmisión sexual $n$ (\%) & $6(43)$ & $5(36)$ & $2(14)$ & $11(79)$ & $2(14)$ \\
\hline & Anticoncepción de emergencia n (\%) & $4(29)$ & $5(36)$ & $3(21)$ & $10(71)$ & $1(7)$ \\
\hline & Pruebas de embarazo n (\%) & $4(29)$ & $4(29)$ & $2(14)$ & $11(79)$ & $6(43)$ \\
\hline & Interrupción del embarazo n (\%) & $6(43)$ & $7(50)$ & $3(21) *$ & $11(79)$ & $5(36)$ \\
\hline & Atención prenatal n (\%) & $3(21)$ & $5(36)$ & $2(14)$ & $11(79)$ & $5(36)$ \\
\hline & Atención del parto n (\%) & $2(14)$ & $5(36)$ & $1(7)$ & $12(86)$ & $6(43)$ \\
\hline & Atención del postparto n (\%) & $3(21)$ & $4(29)$ & $2(14)$ & $11(79)$ & $4(29)$ \\
\hline & Salud menstrual n (\%) & $6(43)$ & $5(36)$ & $2(14)$ & $9(64)$ & $3(21)$ \\
\hline & $\begin{array}{l}\text { Atención a sobrevivientes de } \\
\text { violencia sexual } \mathrm{n}(\%)\end{array}$ & $10(71)$ & $10(71)$ & $9(64)$ & $12(86)$ & $9(64)$ \\
\hline
\end{tabular}

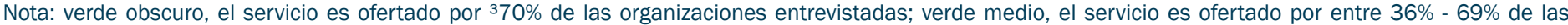
organizaciones entrevistadas; verde claro, el servicio es ofertado por $<36 \%$ de las organizaciones entrevistadas.

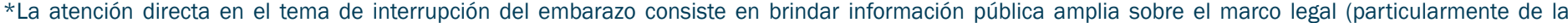

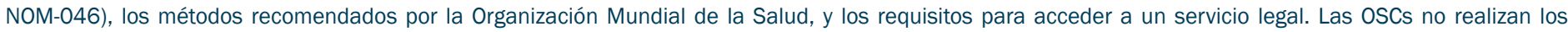

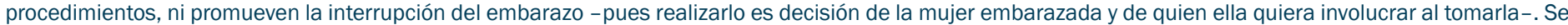
diferencia de “difusión de información” en tanto los temas cubiertos son más amplios en esta categoría. 
Gráfica 2. Porcentaje de organizaciones que cuentan con personal de salud en sus instalaciones. Cludad de México, 2020.

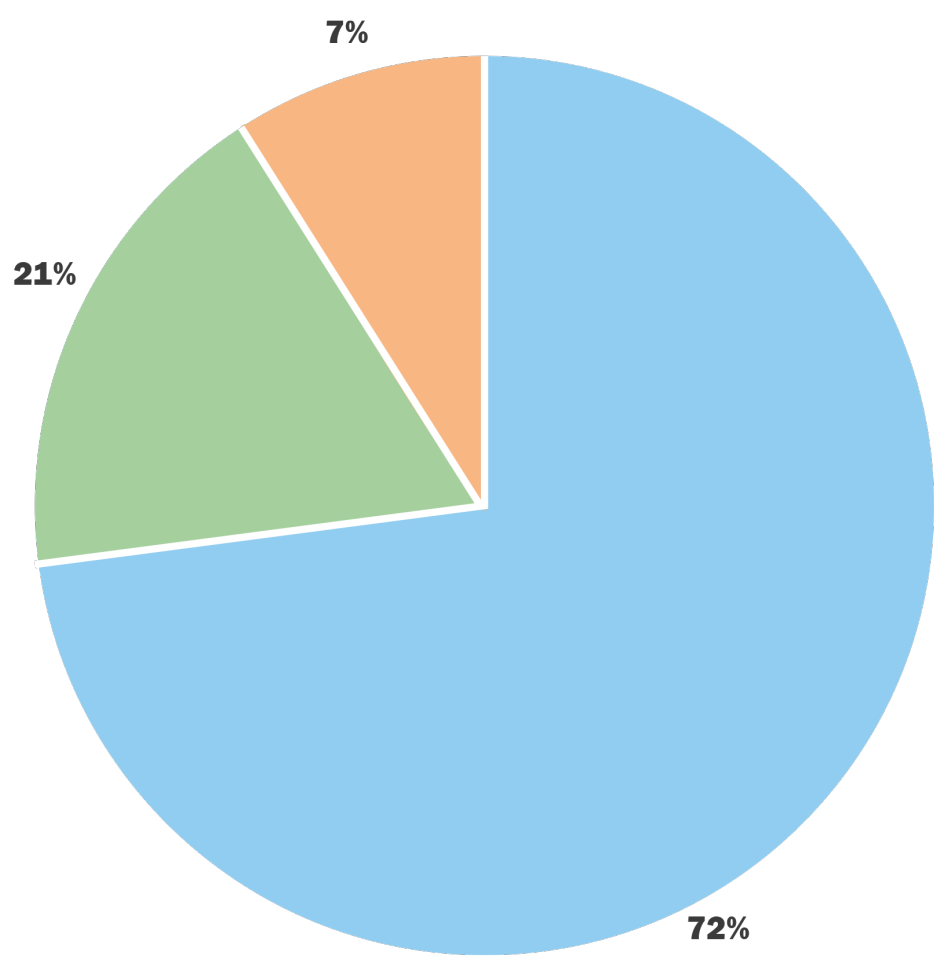

Sin personal de salud

Médica/o de base

Sin respuesta

\section{Principales barreras para la atención en} SSyR a mujeres migrantes

En las entrevistas con organizaciones de la sociedad civil en Ciudad de México se identificaron múltiples barreras para que las mujeres migrantes pudieran acceder a los servicios de SSyR durante su estancia o tránsito por el estado. En el caso del presente estudio, las barreras para el acceso a la salud fueron clasificadas en cuatro categorías:

1. Normativas: relacionadas con la legislación, políticas y normatividad que aplican tanto a la población migrante (marco restrictivo para la atención), como a las instituciones de salud;

2. Institucionales: impedimentos estructurales de la institución de salud que brinda o debería brindar los servicios;

3. Organizacionales: impedimentos estructurales de la organización de la sociedad civil que brinda los servicios; e

4. Individuales: obstáculos derivados de la condición socioeconómica, cultural y subjetiva de las personas migrantes (ver Tabla 2).
Las barreras normativas percibidas por las organizaciones fueron 1) la desaparición del Seguro Popular y la falta de claridad sobre las reglas de operación del Instituto de Salud para el Bienestar (Insabi); 2) falta de perspectiva de género e intercultural bien integrada en la normativa para migrantes, pues, por ejemplo, es difícil obtener una respuesta positiva a la solicitud de la condición de persona refugiada por razones de violencia de género y familiar; 3) el método de asignación de presupuesto a salud invisibiliza las necesidades de las unidades médicas para atender a toda la población en su territorio, pues se distribuye de acuerdo con censos poblacionales en los que la población migrante no es contabilizada.

Con respecto a las instituciones de salud, se identificaron barreras 1) administrativas, ya sea por desconocimiento de las normas existentes ${ }^{3}$ o por una aplicación discrecional de las mismas. Para la atención de salud, se solicita, por ejemplo, comprobante de domicilio; visa humanitaria; además se interponen pasos burocráticos en general; o se desconocen papeles oficiales expedidos por la Comar. Las organizaciones reportaron que los requisitos varían por unidad médica, lo que significa que la atención depende de los reglamentos internos de las unidades o de conocer a alguien que facilite el acceso. Por ello, como se dijo anteriormente, la canalización o acompañamiento parecen requisitos para la atención. También, se señalaron otras barreras como 2) las actitudes discriminatorias, xenófobas y poco sensibles hacia la población migrante; 3) dificultad para encontrar servicios de especialidad médica y acceder a ellos (referencia); 4) falta de personal médico específico para SSyR como parteras; y 5) la falta de intérpretes en las unidades de salud que atienden a población migrante.

Las barreras relativas a las organizaciones de la sociedad civil entrevistadas fueron 1) falta de conocimiento sobre otras OSCs para canalizar a las mujeres; 2) dificultad para tratar temas de SSyR, sobre todo aborto (especialmente en las organizaciones religiosas); 3) ausencia o insuficiencia de infraestructura (espacios) para el alojamiento exclusivo de mujeres; 4) falta de personal capacitado en la atención psicológica a personas que vivieron violencia sexual; así como 5) recursos insuficientes para tener insumos para la atención de emergencia (ej. anticoncepción de emergencia). Las organizaciones reconocen también la limitación en su capacidad para dar seguimiento a la población, dada su característica de movilidad (ej. manejo psicológico profundo para mujeres que vivieron violencia sexual).

Las barreras individuales para la búsqueda de atención 
que identificaron las organizaciones entrevistadas fueron 1) desconocimiento que las mujeres migrantes tienen de sus derechos y la forma de exigirlos, pues piensan que no tienen derecho a la salud; 2) aspectos culturales que impiden a las mujeres buscar ayuda debido a tabús, creencias y desconocimiento sobre la SSyR, y eso complica el abordaje del tema por parte de las organizaciones, al igual que el machismo como limitante para hablar de violencia, anticoncepción o planificación familiar, sobre todo para las mujeres que viajan con sus parejas -además, muchas veces las mujeres no reconocen que viven violencia-.

También, debido a que en Centroamérica la legislación del aborto es altamente restrictiva y penalizada, muchas mujeres no contemplan la posibilidad de abortar (probablemente porque han internalizado el estigma o porque asumen la imposibilidad de hacerlo). Algunas mujeres creen también que un embarazo facilitará el asilo en EE.UU., y buscan transitar por México lo más rápido posible, sin buscar atención. ${ }^{4}$ 3) EI idioma para quienes no hablan castellano sino una lengua indígena u otra distinta al español también representa una barrera para buscar información y atención. Finalmente, 4) los costos de transporte y medicamentos son altos para las migrantes y por tanto una barrera para la atención a su salud.

\section{coVID-19, mujeres migrantes y acceso a servicios de SSYR}

En general, y de acuerdo con el reporte de las organizaciones entrevistadas en los siete estados que son parte de este estudio $^{5}$, se identificó que la pandemia por COVID-19 tuvo los siguientes efectos en las OSCs y en la población migrante, particularmente en las mujeres y su acceso a servicios de SSyR:

- Reducción de unidades de salud y servicios disponibles. La reconversión de clínicas y hospitales a Centros COVID y la atención restringida a casos de emergencia, limitó de manera significativa la atención en las unidades médicas, ya sea por retraso o negación de la atención.

Tabla 2. Resumen de principales barreras para que las mujeres migrantes accedan a los servicios de salud en Ciudad de México, 2020.

\begin{tabular}{|c|c|}
\hline Clasificación & Principales barreras \\
\hline Normativas & $\begin{array}{l}\text {-Desconocimiento de operación del Insabi } \\
\text {-Falta de perspectiva de género e intercultural } \\
\text {-Recursos insuficientes porque la población migrante no es contabilizada }\end{array}$ \\
\hline Institucionales & $\begin{array}{l}\text {-Administrativas, por desconocimiento de las normas existentes o por aplicación } \\
\text { discrecional de las mismas } \\
\text {-Actitudes discriminatorias, xenófobas y poco sensibles hacia la población migrante } \\
\text {-Dificultad para encontrar y acceder a servicios de especialidad médica } \\
\text {-Falta de personal médico, como parteras } \\
\text {-Falta de intérpretes }\end{array}$ \\
\hline Organizacionales & $\begin{array}{l}\text {-Desconocimiento de otras OSCs para canalizar } \\
\text {-Dificultad para tratar temas de SSyR en OSCs religiosas } \\
\text {-Infraestructura } \\
\text {-Falta de personal de psicología de base } \\
\text {-Falta de recursos para adquisición de insumos }\end{array}$ \\
\hline Individuales & $\begin{array}{l}\text {-Desconocimiento de los propios derechos } \\
\text {-Aspectos culturales como tabús y machismo } \\
\text {-Idioma } \\
\text {-Costo de los servicios }\end{array}$ \\
\hline
\end{tabular}


- Cierre temporal y/o parcial de albergues. Incluso aquellos que se mantuvieron abiertos dejaron de recibir a personas, ya que las y los migrantes no acudían porque escucharon que estaban cerrados.

- Disminución de recursos en las OSCs para prestar la atención. Con la pandemia hubo una reducción de recursos recibidos a través de donativos, además de tener una baja en el número de personas voluntarias, $\mathrm{y}$ del personal en general para brindar servicios y atención, incluyendo a personal médico.

- Permanencia prolongada de migrantes en los lugares, al no poder seguir su viaje. El cierre de oficinas gubernamentales - como la Comar- afectó a aquellas personas que esperaban la resolución a su solicitud de condición de refugiada u otro trámite similar, y les obligó a no salir de la entidad donde iniciaron el procedimiento.

- Afectaciones a la salud mental de las personas migrantes. Incremento de las preocupaciones relacionadas con sus procesos frente a autoridades migratorias, y/o por el confinamiento en albergues.

- Pérdida de trabajos y recursos económicos. Con el cierre de establecimientos comerciales hubo pérdida de empleos que también afectó a personas migrantes, pues se enfrentan a la imposibilidad de laborar y tener el ingreso para solventar sus necesidades básicas.

- Miedo a asistir a las unidades médicas. Las personas migrantes tienen temor a contagiarse si asisten a las unidades médicas, por lo que retrasan o desisten de la atención.

\section{CONCLUSIONES Y ÁREAS DE OPORTUNIDAD ${ }^{6}$}

Las posibilidades para mejorar la atención de la salud sexual y reproductiva de las mujeres son amplias, desde el fortalecimiento del sistema de salud público que garantice el acceso a estos servicios, hasta apoyando a las organizaciones y albergues que están dando acceso o facilitando el mismo. Si bien las OSCs no son responsables garantes de brindar servicios de salud, han demostrado ser clave para contribuir a este propósito. En el campo en que se enfocó el estudio (SSyR de las mujeres migrantes), el rol de las OSCs es particularmente relevante, no sólo por ser muchas veces el primer punto de contacto, sino porque la confianza que ganan con su trabajo les habilita para tratar estos temas considerados de mayor intimidad-. La atención a mujeres que han vivido violencia sexual (sobre todo en cuanto a su salud mental) es la necesidad más reconocida y atendida de diferentes formas por las organizaciones que trabajan con esta población en Ciudad de México.

El Estado, a través de sus instituciones, tiene la responsabilidad de garantizar que los servicios de salud se brinden de forma oportuna a las mujeres migrantes. El apoyo a la continua capacitación de servidores públicos sobre los derechos de personas migrantes en México (y sobre lo que implican los cambios en políticas públicas) puede fortalecer la viabilidad de las rutas de acceso a servicios de salud.

Para el sector público de salud, las organizaciones identificaron áreas de oportunidad y responsabilidad relacionadas con asignar un presupuesto adecuado que garantice las necesidades de insumos y medicamentos suficientes y gratuitos para las usuarias. Este financiamiento debe corresponder al volumen de población que las unidades de salud atienden, y deben de contabilizar a la población migrante indocumentada (contemplar a esta población en los censos que se realizan). En este sentido, los sistemas de información deben incluir datos sobre población extranjera migrante atendida en los diferentes servicios para monitorear sus necesidades.

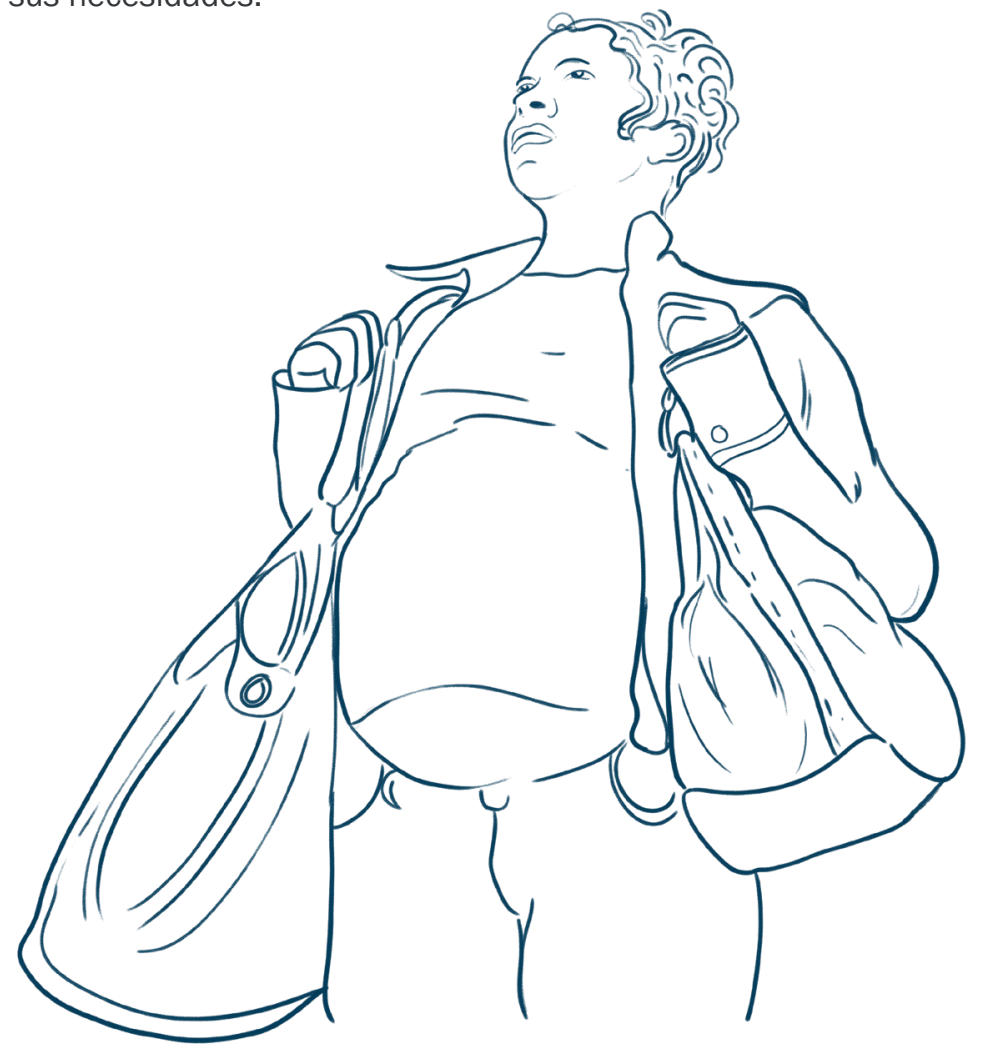


Las organizaciones destacaron también la urgencia de generar y difundir información clara sobre el funcionamiento del Insabi y el proceso que las unidades de salud deben de seguir para garantizar el derecho a la salud de la población migrante indocumentada. Promover un enfoque sensible y especializado entre autoridades y el personal de salud para la atención a población en situación de movilidad, además de implementar dinámicas efectivas para romper prejuicios sobre esta población. Fortalecer la rectoría del estado para permitir únicamente la difusión de información validada por la Secretaría de Salud (esto sobre todo en relación con la interrupción del embarazo, pues hay campañas de desinformación en las cercanías de las unidades médicas que prestan el servicio en la Ciudad de México).

Por su parte, las organizaciones de la sociedad civil pueden, entre otras cosas, ayudar a monitorear las necesidades de SSyR de esta población; brindar información/educación de calidad sobre la SSyR (con base en las necesidades identificadas), y sobre los pasos para ejercer su derecho a la salud; identificar (y educar también sobre) síntomas y señales de riesgo que indican que una mujer debe de ser atendida de urgencia; y establecer mecanismos formales de referencia a unidades de salud correspondientes con el tema a tratar.

Las OSCs que trabajan exclusivamente con la población migrante en la Ciudad de México, reconocen para sí la oportunidad de fortalecer el sistema de alianzas para la referencia de mujeres migrantes con necesidades de SSyR. La perspectiva de género es reconocida como insuficiente en esta comunidad de organizaciones. La alianza con OSCs feministas que manejan los temas de SSyR y/o con los servicios de salud estatales puede mejorar la información, consejería y acceso a los servicios que las mujeres requieren.

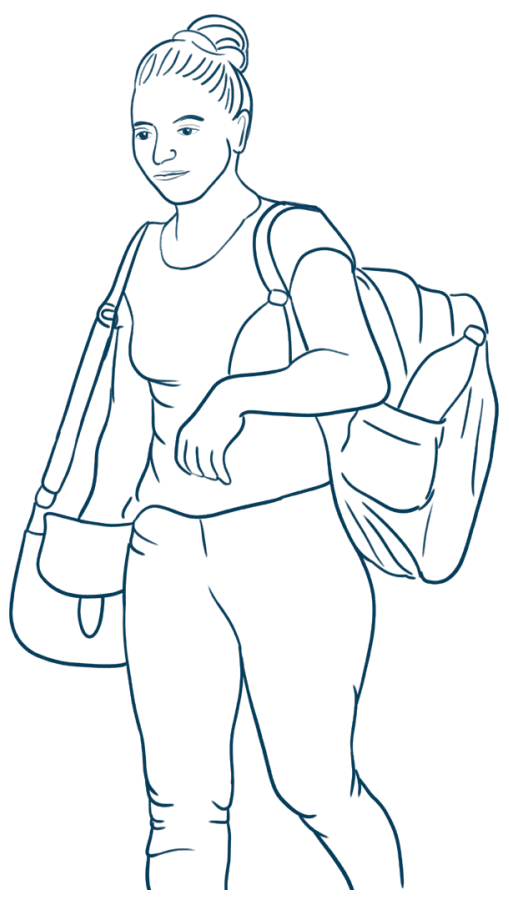

Reforzar el conocimiento sobre promoción, prevención y signos y síntomas de riesgo con respecto a métodos anticonceptivos, anticoncepción de emergencia, ITS, pruebas de embarazo, interrupción del embarazo, atención prenatal, atención del trabajo de parto y atención del postparto, salud menstrual y primeros auxilios para la atención a personas que vivieron violencia sexual. Por otra parte, las organizaciones feministas no refieren generar información o discurso dirigido especialmente a mujeres migrantes en tránsito o viviendo de forma temporal en México, ni a organizaciones que trabajan con ellas. Generar comunicación y mensajes dirigidos a esta población es importante sobre todo si el tabú o desconocimiento sobre estos temas impide que las mujeres (y/o quienes trabajan con ellas) busquen información o atención de manera más expresa. De igual forma, se recomienda adecuar los requisitos de atención que algunas de las organizaciones tienen (como ser necesariamente acompañadas) a la circunstancia y necesidades de las mujeres migrantes y solicitantes de asilo.

Incluir información que visibilice la SSyR en los materiales que generan las organizaciones puede facilitar que las mujeres le den también importancia. Las propuestas de las organizaciones incluyen hacer llegar información en idiomas distintos al castellano (ej. lenguas indígenas y creole), así como información para las personas con baja alfabetización por radio comercial y comunitarias. La difusión de información sobre servicios de salud a través de pegatinas y carteles en lugares concurridos han sido estrategias consideradas exitosas por las organizaciones entrevistadas.

Otra área de oportunidad es la de contar con un sistema de información que les permita el monitoreo de la población a la que atienden y los problemas de salud (en general y en particular, SSyR) presentados $\neg$-entre otras temáticas relacionadas con el respeto a sus derechos humanos-. La finalidad del monitoreo es la de sistematizar e identificar las necesidades más apremiantes de esta población y cómo se van transformando en el tiempo. Esto ayudará a las organizaciones a contar con información para hacer incidencia con las instituciones de salud gubernamentales, y recomendar la priorización de esfuerzos y recursos en el corto, mediano, y largo plazo. El sistema de información se puede generar también con el apoyo de organizaciones de la sociedad civil nacionales y/o internacionales. Con el fin de hacerse de recursos para la creación de este sistema de información seguro y compartido entre las redes de apoyo a migrantes, se puede insistir a través de la incidencia con quienes financian trabajo en este campo.

Relacionado con lo anterior, una necesidad sentida al interior 
de las organizaciones es el acceso a financiamiento para contratar personal, particularmente aquel especializado en salud mental (psicología, psiquiatría) para la atención a mujeres que han vivido violencia sexual. El acceso a insumos como la anticoncepción de emergencia también se puede ver restringida por la falta de recursos financieros. El apoyo gubernamental estatal y federal, de agencias internacionales, y de personas individuales es necesario para cubrir esta brecha; y se hace un llamado a estos actores a no desestimar el papel que las organizaciones cubren para procurar el bienestar de las personas migrantes. Por otro lado, el acceso a servicios de salud especializados (como oncología y ginecobstetricia) es difícil incluso si las mujeres son acompañadas por las organizaciones. Esto se debe en gran parte a la fragmentación de servicios y a que las referencias al segundo y tercer nivel de atención en instituciones públicas de salud, toma tiempo dentro del sistema. La movilidad de las mujeres dificulta este seguimiento, por lo que al corto plazo, las alianzas con especialistas que puedan trabajar probono en la atención a esta población puede ser una alternativa. El desarrollo de la telesalud abonará a la posibilidad de dar seguimiento, y el involucramiento de las mujeres migrantes en el desarrollo de estos proyectos será clave.

A pesar de las limitaciones del estudio (como contar sólo con el reporte de las OSCs, y no con la perspectiva ni de las mujeres ni del sistema de salud local), la investigación muestra importantes áreas de oportunidad para lograr la atención a la SSyR de las mujeres migrantes. Investigaciones futuras deberán de indagar directamente sobre las necesidades de SSyR de mujeres, adolescentes y niñas, y las necesidades del sector salud para poder prestar la atención. La investigación operativa puede mejorar el funcionamiento de las OSCs como puentes para acercar a las mujeres a los servicios de salud, y apoyar a las unidades médicas a mejorar sus protocolos, así como a entender por qué ciertos esfuerzos - de capacitación, por ejemplo-, pueden no estar dando el resultado esperado. La salud sexual y reproductiva de las mujeres migrantes en tránsito por México es un derecho. Tomar conciencia de que las necesidades de salud de las mujeres son minimizadas, incluso por ellas mismas, es uno de los retos hacia la equidad de género. Facilitar y garantizar el cuidado a su salud forma parte de los esfuerzos para lograr el respeto a los derechos humanos.

Agradecemos el esfuerzo realizado por el equipo de investigación durante los meses de trabajo, así como la gran disposición que las personas entrevistadas tuvieron para compartirnos su experiencia en medio de la pandemia de SARS-CoV-2, y por su compromiso constante con las mujeres migrantes.

El presente estudio es evidencia de que las organizaciones de la sociedad civil locales e internacionales trabajan en primera línea para defender, promover, y apoyar el alcance de los derechos de las personas migrantes -incluso coadyuvando en el acceso a servicios de salud sexual y reproductiva-.

El reconocimiento a ellas y a su labor va acompañado de un llamado a la solidaridad para impulsar su financiamiento y generar condiciones que permitan su desarrollo y el de sus colaboradoras/es.

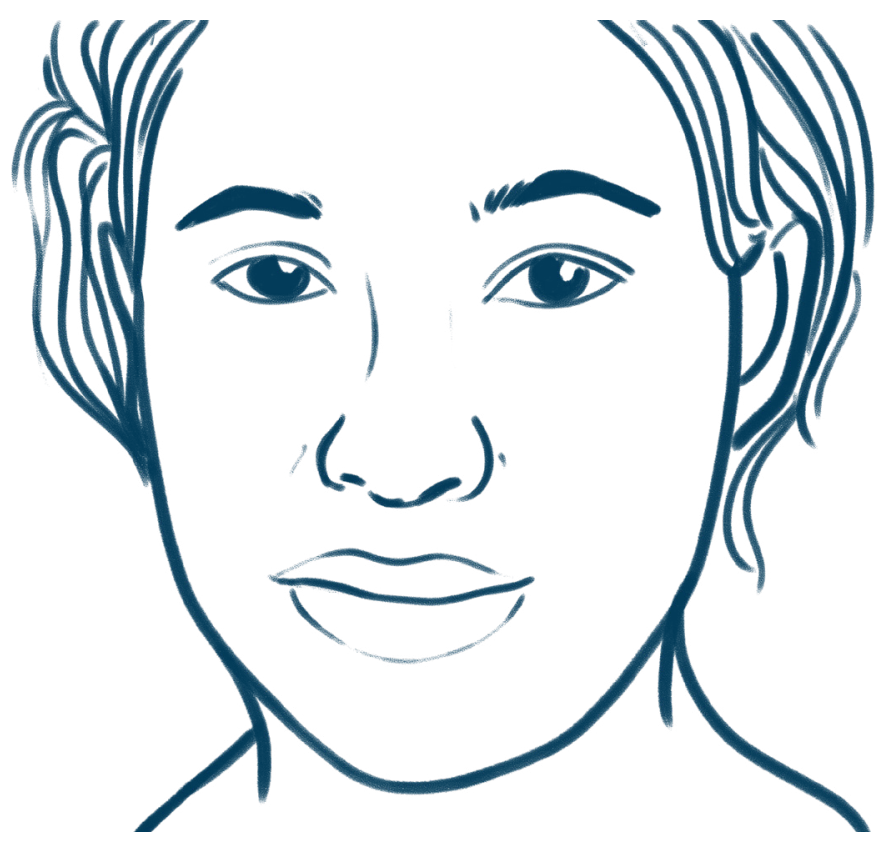

Population Council México. 2021. Atención de la salud sexual y reproductiva de mujeres migrantes: Un mapeo de actores de la sociedad civil en México. Resultados principales para el estado de Chiapas. Ciudad de México, México: Population Council. 
1. ACNUR. Encuesta sobre la Población Refugiada en México. México, 2017. Disponible en: https://www.acnur.org/publications/pub_inf/5ddff2ca4/encuesta-sobre-la-poblacion-refugiada-en-mexico-enpore-2017.html

2. Comar. Boletín estadístico de solicitantes de refugio en México de la Comisión Mexicana de Ayuda para Refugiados. SEGOB. México, 2018. Disponible en: https://www.gob.mx/cms/uploads/attachment/file/427549/COMAR2018.pdf

3. Constitución Política de la Ciudad de México. México, 2017. Disponible en: http://www.infodf.org.mx/documentospdf/constitucion_cdmx/Constitucion_\%20Politica_CDMX.pdf

4. Gobierno de la Ciudad de México. Diagnóstico y Programa de Derechos Humanos de la Ciudad de México. CDMX, 2016.

5. Oxfam (11 de mayo de 2018). ¿Qué es una ciudad santuario como la CDMX? Disponible en: https://www.oxfammexico.org/ historias/\%C2\%BFqu\%C3\%A9-es-una-ciudad-santuario-como-la-cdmx\#: :text=Son\%20lugares\%20que\%20se\%20declaran,el\%20acompa\%C3\%B1amiento\%20legal\%20de\%20las 